\title{
5G system throughput performance evaluation using Massive-MIMO technology with Cluster Delay Line channel model and non-line of sight scenarios
}

\author{
John Baghous
}

\begin{abstract}
The fourth-generation system for mobile cellular communications (4G) has achieved great developments. The main problem here is that, with the passage of time and technical development, the need for new applications and services has emerged, and thus we need a new system that supports these matters in addition to the problems and limitations. One of the main challenges that the $4 \mathrm{G}$ system suffers from is the ability to support a larger number of devices, low latency, working in real time, provide greater capacity, in addition to providing a high data rate (bit rate) - hence 4G stands unable to support many new applications. This is what made researchers aspire to overcome these problems or reduce their impact to the maximum extent and this is what we expect to achieve in the new generation system (5G). In this research, a presentation was made of the $5 \mathrm{G}$ system regarding with one of its most important techniques (Massive MIMO technology), clarification of some concepts related to the study such as throughput and NLOS (Non-Line of Sight), as well as the channel model used. The results of the experiments were presented with the discussion.
\end{abstract}

Index Terms-(5G mobile networks), Massive MIMO technology, 5G channel models, NLOS scenario, 5G Throughput

\section{INTRODUCTION}

Although wireless cellular technologies have been upgraded to the fourth generation, it still suffers from some problems, as it is unable to meet the requirements of many new use-cases. For example, the $4 \mathrm{G}$ network cannot handle massive mobile broadband requirements, and it is difficult to achieve Deviceto-Device communication anywhere. It is also unable to support HD video transmission, high-quality audio, augmented reality, virtual reality and other services, so the $4 \mathrm{G}$ system has left some important unresolved problems, such as limited bandwidth, unlimited peripheral increase, limited data rate and more [1]. Therefore, new-generation wireless networks must be optimized to meet the demands of increasing data rate, improving capacity, reducing latency and improving quality of service. With the increasing demand from users, the $4 \mathrm{G}$ network will be extended (and then maybe replaced) by the $5 \mathrm{G}$ network with the help of some advanced technologies such as Massive MIMO, Device-to-Device

John Baghous is with Faculty of Engineering, Damascus University (e-mail: john0baghous@gmail.com). communication, millimeter waves connections, beam division multiple access and others [2]. The goals of the $5 \mathrm{G}$ cellular communication system are to achieve an end-user data rate 10 to 100 times higher and this is the key here, as it ranges from 1 to $10 \mathrm{Gbps}$ in dense urban environments. The $5 \mathrm{G}$ network may also support higher endpoint density: 5 to 10 times the connected devices in a given area. The energy efficiency in low-power dense machine communications need to improve more than ten times, so it is necessary to introduce new technologies in 5G system to achieve this matter [3]. The Massive MIMO technology attracted great interest in previous years and was considered one of the most promising and most important (radio-related) technologies in the $5 \mathrm{G}$ system by applying a large amount of antennas at the base station that can support many users in the same time frequency domain. It also possesses potential advantages for increasing the efficiency, improving the frequency spectrum, and facing channel fading $[3,4]$.

\section{METHODS AND EXPERIMENTS}

Although mathematical, programming and simulation methods were used in the research behind this paper, but because of the inability to carry out experiments and take practical measurements on the ground, this was sufficed. After verifying the correctness and accuracy of the implementation and based on the mathematical comparison between the results obtained in the simulation and the equations used, this report was written in a summary.

Computers and appropriate software were used to obtain the results. I conducted a performance evaluation of the $5 \mathrm{G}$ system based on a series of comparisons to ascertain the extent of practical investigation of some theoretical issues.

In order to complete the work on this paper, I recommend that realistic measurements be made to compare them with my resulting standardized results to reach results that benefit workers in this field.

\section{The Fifth Generation OF Mobile WiRELESS CELlular COMMUNICATIONS}

The 4G cellular mobile technology has been published and gradual improvements are being made to it, but it has almost reached a state of maturity, so it is necessary to go to the new 
generation [5]. The 5G is the name of the new generation of mobile cellular wireless communications. As expected, this new system will provide high speeds ranging between 10 and $100 \mathrm{Gbps}$ in the future. Moreover, this considered one of the most important strength foundations of this system. In addition to enough capacity that greater than previously and low delay, where the delay time that was provided by $4 \mathrm{G}$ system ranges between 40 and $60 \mathrm{~ms}$, whereas in $5 \mathrm{G}$ the delay time will be between 1 and $10 \mathrm{~ms}$. One of the main technologies that will be used in this system are Massive MIMO, that will have many benefits such as achieving an increase in capacity and throughput, Device-to-Device (D2D) communication, millimeter wave technology and some multi-access technologies such as Beam Division Multiple Access (BDMA).

It also plans to connect all equipment and terminals to the network to obtain what is called Internet of Things (IoT) and after that we are expected to become Internet for everything. Also, some of the connected terminals may need large amounts of data while others need small packets of them. Therefore, the bandwidth of this can be allocated adaptively, including improving the overall capacity of the system. Among the requirements and challenges of the $5 \mathrm{G}$ system are power efficiency, high reliability and availability, large capacity and low delay, given that the system deals with a high bit rate.

There is a major problem related to how to reduce the delay time and to support applications and provide services we need a delay of less than $1 \mathrm{~ms}$ [2]. The $5 \mathrm{G}$ network will not be a single system that relies on a single Radio Access Technology (RAT) similar to previous generations. Further, it is believed that the $5 \mathrm{G}$ network is a "network of networks". That is a heterogeneous system that includes a variety of radio interfaces and protocols frequency bands, access nodes, and different types of networks. This means that the $5 \mathrm{G}$ system will not be a single system that replaces the previous $4 \mathrm{G}$ system, but rather will combine all of the above and what is new. So, one of the main challenges will be the smooth integration between everything old and new [6].

The requirements for this system are expected to be met by the new spectrum that reaches the millimeter wavelength bands and use of the wide channel bandwidth available in the millimeter bands. Although the demand for data is increasing significantly, the usage patterns of this system are not only limited to the pattern of mobile broadband use, but it is expected to support a variety of usage scenarios classified into three broad categories [7]:

- Enhanced mobile broadband (eMBB)

- Ultra-reliable and low latency communications (URLLC)

- Massive machine type communications (mMTC)

In order to understand the engineering challenges facing this system concretely, and plan to meet them, it is necessary first defining their requirements, but it must be emphasized that it is not necessary to meet all these elements at one time, as different applications will put different requirements on performance. The following elements are the system's major requirements in each major dimension that must be met in certain situations: data rate, delay, power and cost, density [5].

The need for a higher data rate in all areas is receiving one of the greatest interests and this is discussed in this paper. Our view is that the improvements in $5 \mathrm{G}$ system will be achieved through combined gains in three categories [5]:

A. Increase density greatly to improve the spectral efficiency of the area and increase the number of active nodes within one area and frequency.

B. Increase the bandwidth, mainly by moving towards the wide spectrum and its capabilities, and also by making better use of the unlicensed spectrum of Wi-Fi in the $5 \mathrm{GHz}$ range to obtain a larger frequency spectrum.

C. Increase spectral efficiency, primarily by advancing the MIMO rank, to achieve a higher throughput per channel and per node.

Using wider frequency range between certain number of nodes will not necessarily increase the achievable bandwidth. Other ideas not included in the above categories such as managing frequency interference through cooperation of base stations may contribute to the improvements, but the increase in capacity should come from the ideas in the above categories. One of the new things in the system is the issue of millimeter waves, whose range is between 30 and $300 \mathrm{GHz}$, with wavelengths ranging from 1 to $10 \mathrm{~mm}$. This field of millimeter wave spectrum has not been used for a long time because, until recently, it was considered unsuitable for mobile communications due to its rather subtle and complex propagation characteristics, including high path loss, atmospheric and rain absorption, low diffraction around obstructions and weak penetration through various objects, due to strong phase noise. However, with progress and technical development, work is in progress to solve most of the cost and other related problems [5].

\section{The MASSIVE MIMO TECHNOLOGY}

The Multi-user Multiple-Input Multiple-Output (MIMO) technology provides significant advantages over traditional Point-to-Point MIMO technology [15] as it offers improvements in several aspects: increasing the data rate, enhancing reliability, improving power efficiency, reducing interference [8].

Due to the wide use of multimedia application services such as voice, writing, pictures, videos, Internet access, etc. In recent years, the demand for the rapid transmission of information and the reliability of communications through wireless communication systems has increased greatly, and to overcome these limitations we are going to use multiple antennas at the same time in transmission and reception. Transmission systems take advantage of the spatial dimension in order to transmit information. This technology is called Massive MIMO or Wide Field MIMO as it allows us to improve the throughput and performance of wireless links [9, 16]. This technology provides significant support to the 
$5 \mathrm{G}$ system throughput performance evaluation using

Massive-MIMO technology with Cluster Delay Line

channel model and non-line of sight scenarios

system through the use of a large number of antennas with a time-dividing process. Multiple antennas help to focus power in consistently smaller areas to provide significant improvements in throughput, low latency, and radiated power efficiency [6]. Whereas the expected throughput depends on the propagation environment which provides orthogonal channels converging to the user terminals [8].

In MIMO technology, communication takes place in two ways: spatial diversity and spatial multiplexing. In spatial diversity, the same data travels over different paths and the data are received by multiple antennas and processed. Using this technology, we can improve the reliability of the link. The other technique is spatial multicast, where the data is divided into small parts and each part is transmitted through a different path and thus the transmission speed is increased at the expense of less reliability [2].

The MIMO system generally consists of a number of transmitting $(\mathrm{M})$ and receiving $(\mathrm{N})$ antennas and the communication channel through which the signal passes. Thus, the general equation for this technique is given by the equation (1) [2]:

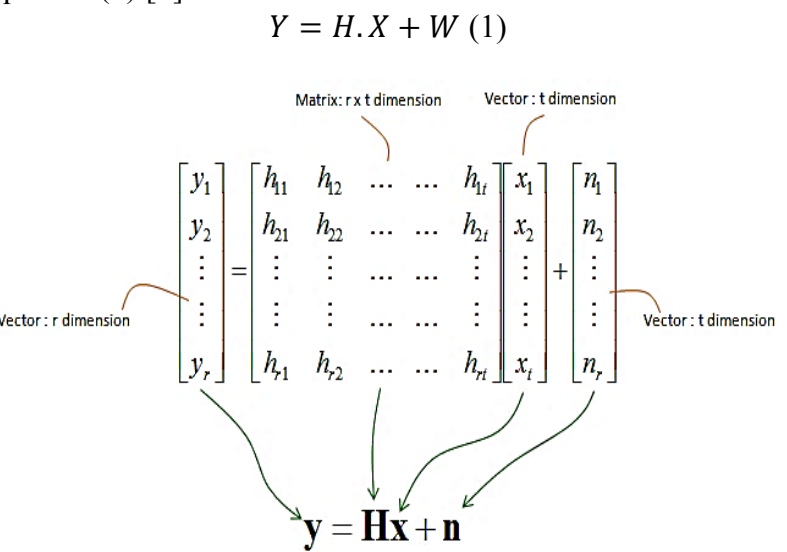

Fig. 1. A Massive MIMO equation.

Where: $\mathrm{Y}=\mathrm{N} \times 1$ receiver matrix, $\mathrm{H}=\mathrm{N} \times \mathrm{M}$ channel matrix, $\mathrm{X}=\mathrm{M} \times 1$ transmitter matrix and $\mathrm{W}$ is the noise. This is illustrated in Figure 1.

We have two scenarios for these networks, internal and external. For the external, the user terminal will communicate with the antennas distributed at the cell site, while the internal will be in cooperation with Wi-Fi technology, optical

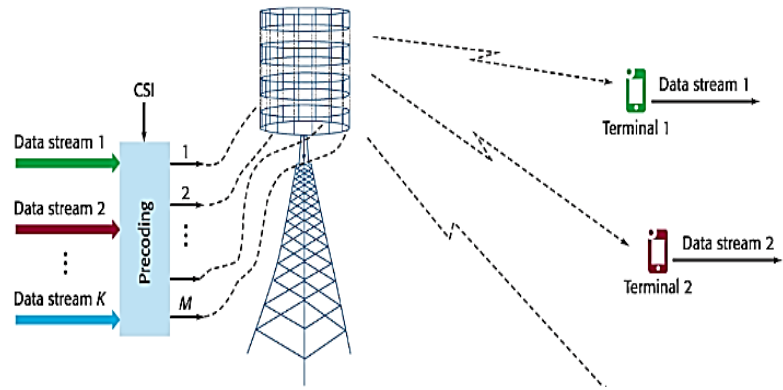

Fig. 2. Massive MIMO technology in the case of the downlink. communications, and millimeter wave technology [2] Figure 2 shows the Massive MIMO technology in a downlink condition.

\section{THE IMPORTANCE OF IMPROVING THE THROUGHPUT}

There are two basic facts: firstly, the demand for wireless productivity will always increase and secondly: the amount of available electromagnetic frequency spectrum will not increase. Taking into consideration that wireless communications are radically different from optical fiber communications, as more fibers can always be manufactured and put in place, and there is no doubt that any future optical demand will always be met. In contrast, there is no easy solution to wireless throughput [10]. The throughput is generally given to a given area by equation (2) [11]:

$$
\begin{gathered}
\text { Area throughput }\left(\frac{b p s}{\mathrm{~km}^{2}}\right)=B W(\mathrm{~Hz}) \times \text { Cell density }\left(\frac{\text { cells }}{\mathrm{km}^{2}}\right) \times \\
\text { Spectral efficiency }\left(\frac{\frac{b p s}{\mathrm{~Hz}}}{\text { cell }}\right)(2)
\end{gathered}
$$

Our basic wireless problem arises in the physical layer of how to reliably and uniformly provide increased overall wireless throughput across a given region [10].

The previous simple relationship, equation (2) shows that there are three main components that can be improved to achieve higher throughput $[11,10]$ :

1. More bandwidth can be allocated to $5 \mathrm{G}$ services.

2. The network can be condensed by adding more cells with access points operating independently.

3. The efficiency of data transmission (per cell and for a specified range of bandwidth) can also be improved and the use of multiple antennas at both the transmitting and receiving ends.

In this paper, Throughput has been calculated based on the bit rate equation of the $5 \mathrm{G}$ system shown in equation (3) [14]:

$$
10^{-6} \sum_{j=1}^{J}\left(\begin{array}{c}
\text { Data Rate (Mbps) }= \\
v_{\text {Layers }}^{(j)} \cdot Q_{m}^{(j)} \cdot f^{(j)} \cdot R_{\max } \cdot \frac{N_{P R B}^{B W(j), u} \cdot 12}{T_{s}^{u}} \cdot \\
\left(1-O H^{(j)}\right)
\end{array}\right)
$$

where $\mathrm{J}$ is the number of aggregated component carriers in a band or band combination; $\mathrm{R}_{\max }=948 / 1024 ; v_{\text {layers }}^{(j)}$ is the maximum number of layers; $Q_{m}^{(j)}$ is the maximum modulation order and takes the following values ( 2 for QPSK, 4 for 16QAM, 6 for 64-QAM, 8 for 256-QAM); $f^{(j)}$ is the scaling factor, the scaling factor can take the values $1,0.8,0.75$, and 0.4. $\mu$ is the numerology (as defined in 3GPP TS 38.211) and can takes values from 0 to $5 . T_{S}^{\mu}$ is the average OFDM symbol duration in a subframe for numerology. $N_{P R B}^{B W(j), \mu}$ is the maximum $\mathrm{RB}$ allocation in bandwidth. $B W^{(j)}$ with 
$5 \mathrm{G}$ system throughput performance evaluation using

Massive-MIMO technology with Cluster Delay Line channel model and non-line of sight scenarios

numerology $\mu$ where $B W^{(j)}$ is the UE supported maximum bandwidth. $O H^{(j)}$ is the overhead and takes the following values:

- $\quad$ FR1 frequency range: DL: 0.14; UL: 0.08

- $\quad$ FR2 frequency range: DL: 0.18 ; UL: 0.1

\section{Channel Model AND Propagation Scenario}

The Cluster Delay Line (CDL) channel model consists of a number of independent groups of delayed beams wherein each group contains a number of multiple path components that have the same known delay values but differ in departure angles and arrival angles. The beam angle difference may be different from the base station with respect to the mobile terminal and the displacement angles are Laplacian represented for each beam [12]. The CDL model takes into account all factors that affect the signal through the communication channel, in addition to the characteristics of the transmitting and receiving antennas. (Massive MIMO technology in this case.) In addition to the multi-path signal, where in the real environment, the received signal usually consists of a direct path and many paths, these paths differ in number and depend on the interaction between the electromagnetic wave and surrounding obstacles [12][13]. The signal obtained at the receiver (the receiving antenna) corresponds to the sum of these waves that reach the receiver

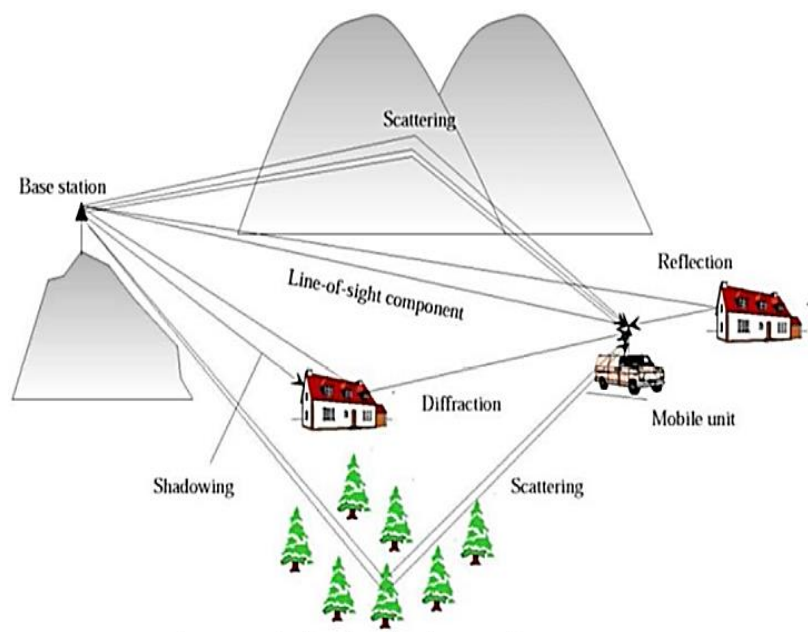

Fig. 3. The multipath propagation scenario.

with different delays [12]. In some environments, such as indoor, the Line-of-Sight (LOS) may not always be available. In this case, the Non-Line-of-Sight (NLOS) pathways allow communication as the signal in this case gets phase and amplitude changes. Figure 3. illustrates the concept of multipath propagation as well as the main propagation phenomena encountered [12].

This model was presented as one of the $5 \mathrm{G}$ models that were presented in 3GPP TR 38.901 version 14.0.0 Release [13]. As it supports a frequency range between 0.5 and $100 \mathrm{GHz}$ and supports channel bandwidth up to $2 \mathrm{GHz}$, the propagation scenarios related to the NLOS are divided into
3 types, namely CDL-A, CDL-B and CDL-C [13]. We have shown that there are differences between the three models in terms of usage scenario [13]. All information such as formulas and tables can be found in [13].

TABLE I

PARAMETERS USED DURING SiMULATION

\begin{tabular}{ll}
\hline \multicolumn{1}{c}{ Parameter } & \multicolumn{1}{c}{ Value } \\
\hline Code Rate & $1 / 2$ \\
Modulation & $16 \mathrm{QAM}$ \\
Subcarrier spacing $(\mathrm{kHz})$ & 30 \\
Resource block & 30 \\
Layers & 2 \\
Number of sending frames & 5 \\
The number of transmitting antennas & $8,16,32,64,128,256$ \\
The number of receiving antennas & $2,4,8,16$ \\
Channel models & CDL-A. B. C \\
Parameter & value \\
Code Rate & $1 / 2$ \\
Modulation & $16 \mathrm{QAM}$ \\
Subcarrier spacing $(\mathrm{kHz})$ & 30 \\
Resource block & 30 \\
\hline
\end{tabular}

\section{IMPLEMENTATION AND RESULTS}

Specific parameters were used during the simulation process, which are shown in Table 1.

\section{PRELIMINARY EXPERIMENTS}

Here we will change the number of transmitting antennas (Tx) related to Massive MIMO technology to the following values: $8,16,32,64,128,256$ and keep the number of receiving antennas (Rx) equal to (2) and measure the extent to which this change affects the throughput performance. These

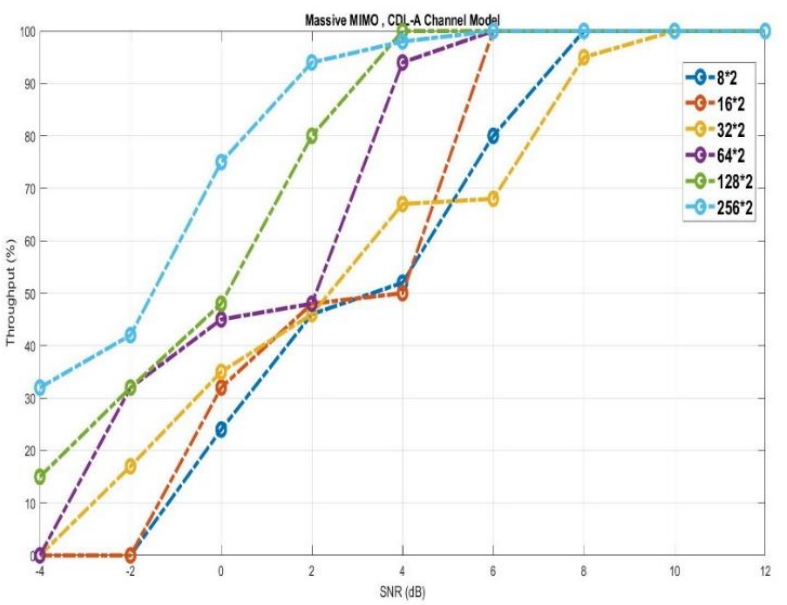

Fig. 4. System throughput with a number of antennas $(8,16,32,64,128$ and $256 \times 2$ ) with (CDL-A) channel model.

experiments in three scenarios for the channel model used CDL-A, B, C will take a relationship between the system throughput and Signal-to-Noise (SNR) value and compare the results. In case CDL-A, we have Figure 4, in case CDL-B, we have Figure 5, and in case CDL-C, we have Figure 6. 
$5 \mathrm{G}$ system throughput performance evaluation using

Massive-MIMO technology with Cluster Delay Line channel model and non-line of sight scenarios

We will divide the notes and discussion on the previous figures into two parts: the first relates to Massive MIMO technology and the second relates to the channel model used.

With regard to the technology used, the increase in the number of transmit antennas array led to improved performance and consequently improved QOS for users.

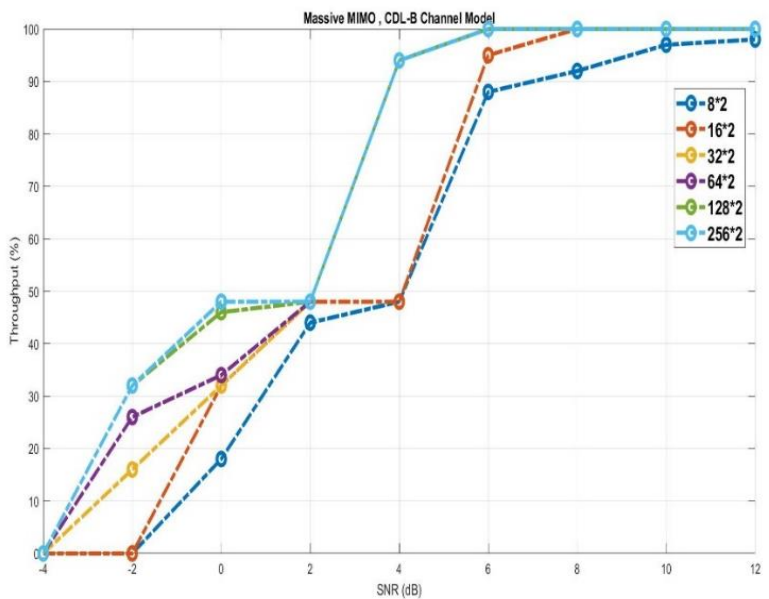

Fig. 5. System throughput with a number of antennas $(8,16,32,64,128$ and $256 \times 2)$ with $($ CDL-B) channel model.

Technically, it can be said that increasing the number of transmit antennas array number contributed to an increase in the number of bits arrive to receiver, including an increase in the bit rate as this process contributes to improving the spectral efficiency of the system, that is, the number of transmitted bits/Hz.

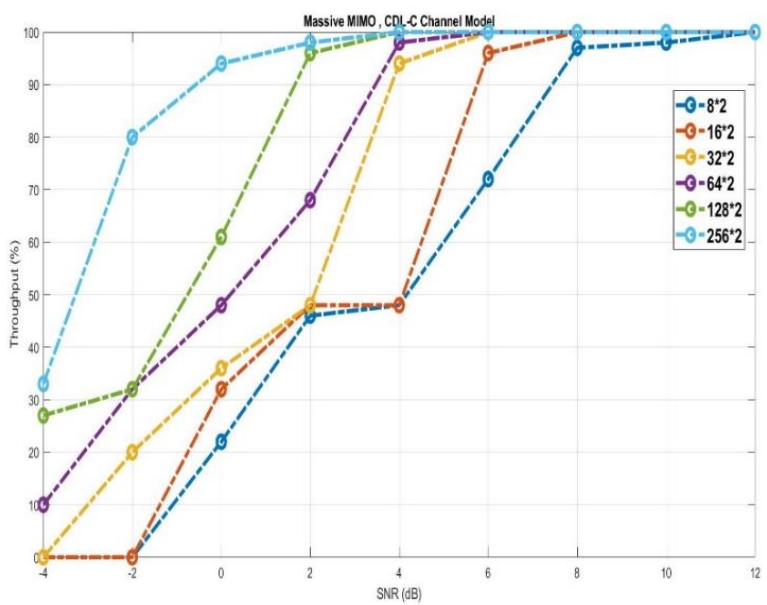

Fig. 6. System throughput with a number of antennas $(8,16,32,64,128$ and $256 \times 2)$ with $($ CDL-C) channel model.

As for the channel models used, we notice from the previous figures that, the better performance of the system was in the case of the CDL-C model, then CDL-A, then CDL-B. As in case of the CDL-C model, the effect of increasing the number of antennas was very clear with the curves, while this clarity decreased in the CDL-A model and decreased further in the CDL-B model.

In general, the advantage of this technique is to obtain higher system throughput at lower SNR values, that is, to obtain good performance under difficult ambient conditions and big noise.

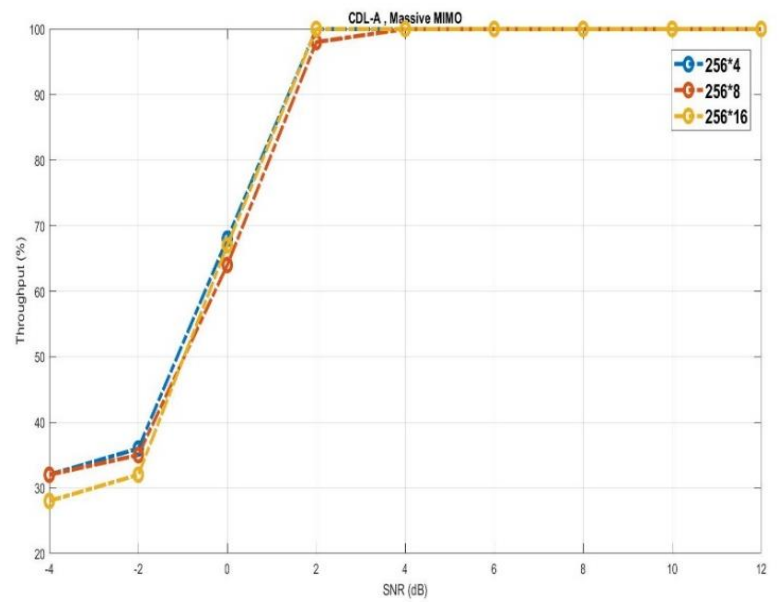

Fig. 7. System throughput with a number of antennas $(256 \times 4,256 \times 8$, $256 \times 16$ ) with (CDL-A) channel model.

\section{INCREASING THE RECEIVER ANTENNAS NUMBER}

In this section, the simulation process is presented to increase the number of receiving antennas while the number of transmitting antennas remains constant with the effect of that on throughput, the amount of bits reached to the user.

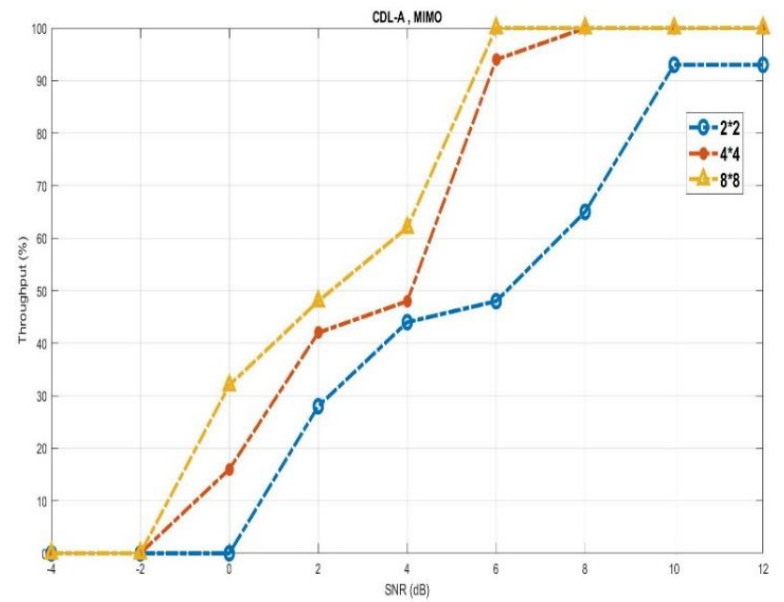

Fig. 8. System throughput with a number of antennas $(2 \times 2,4 \times 4,8 \times 8)$ with (CDL-C) channel model.

As we see in Figure 7, The effect of increasing the number of receiving antennas did not appear clearly and it may appear if we raise the number to greater values up to 256 , and this matter requires an advanced computer with high and modern capabilities and may take a longer time to implement the operation with the increase in the number of antennas. 
$5 G$ system throughput performance evaluation using

Massive-MIMO technology with Cluster Delay Line channel model and non-line of sight scenarios

\section{System Performance When USING MIMO}

In this section, the case of using normal MIMO is presented as we see in Figure 8, which the number of antennas is low and not dense as in the case of the latest technology of MIMO. When comparing this figure with the other previous figures we note that the throughput was non-existent at low SNR values. In addition, the throughput did not reach the upper limit only with an increase in the SNR ratio to high limits unlike cases in which the number of antennas was more.

\section{DISCUSSION}

The previously shown simulations can be divided into three types. In the first case, we demonstrated the effect of increasing the number of transmitting antennas with the constant number of receiving antennas on the system's throughput performance. As is evident, the increase in the number of antennas leads to improved performance by obtaining higher throughput at a lower SNR value. This is a very important improvement because with the increase in the throughput, the spectral efficiency also increases and thus we will have an improvement in the system performance.

In the second case, we increased the number of receiving antennas under the scenario of the CDL-A channel model as an example, and the case showed a convergence in the performance of the throughput curves.

In the third case, we experimented with the use of MIMO technology with fewer antennas at both ends of the communication, in the case of using the CDL-C channel model as an example. In addition, we noticed a clear difference in throughput between this case and the case of Massive MIMO technology. We obtained higher throughput rates in the case of Massive MIMO compared to MIMO case.

\section{CONCLUSION AND FUtURE WORK}

We notice from the previous results that the throughput of the system has improved with the use of Massive MIMO technology. Further, with the improvement of throughput, we will obtain higher values of bit rate at lower SNR values, and we will obtain an improvement in the spectral efficiency. Accordingly, we notice an improvement in the performance of the studied system, and from it, this modern technology will leave its effective impact on the ground and improve the user experience.

In the future, we are looking forward to conduct further experiments on 5G system, in addition to study other parameters and technologies related to this system, and we are looking forward to develop in this regard.

\section{REFERENCES}

[1] Ni, Shanjin. "The Key Technologies in Physical Layer of 5G Wireless Communications". Research Gate. 2017.

[2] Hussain, S. S., Yaseen, S. M., \& Barman, K. "An Overview of Massive MIMO System in 5G". International Science Press, IJCTA, 2016. pp. 4957.
[3] Zheng, K., Leung, V. C., Yang, L. L., \& Chatzimisios, P. "Guest Editorial Special Issue on 5G Wireless Systems with Massive MIMO". IEEE Systems Journal, vol.11, n. 1, 4-6. 2017. DOI: 10.1109/JSYST.2017.2651338.

[4] Liang, W., Wang, Y., Li, B., Wang, W., Sheng, J., Han, Y. \& Kishiyama, Y.. "Ultra-high-Throughput Massive MIMO field-trial over radio computing architecture with peak spectrum efficiency of $79.82 \mathrm{bps} / \mathrm{Hz}$ ". In 2017 IEEE 28th Annual International Symposium on Personal, Indoor, and Mobile Radio Communications (PIMRC) (pp. 1-7). IEEE. 2017, October. DoI: 10.1109/PIMRC.2017.8292309.

[5] Andrews, J. G., Buzzi, S., Choi, W., Hanly, S. V., Lozano, A., Soong, A. C., \& Zhang, J. C." What will 5G be?" IEEE Journal on selected areas in communications, vol. 32, n. 6, 1065-1082. 2014. DoI: $10.1109 /$ JSAC.2014.2328098

[6] Chávez-Santiago, R., Szydełko, M., Kliks, A., Foukalas, F., Haddad, Y., Nolan, K. E. \& Balasingham, I. "5G: The convergence of wireless communications". Wireless Personal Communications, vol. 83, n. 3, 1617- 1642. 2015. DoI: 10.1007/s11277-015-2467-2.

[7] Shafi, M., Molisch, A. F., Smith, P. J., Haustein, T., Zhu, P., De Silva, P., Tufvesson, F., Benjebbour, A. \& Wunder, G. "5G: A tutorial overview of standards, trials, challenges, deployment, and practice". IEEE journal on selected areas in communications, vol. 35, n. 6, 1201-1221. 2017. DoI: 10.1109/JSAC.2017.2692307.

[8] Larsson, E. G., Edfors, O., Tufvesson, F., \& Marzetta, T. L. "Massive MIMO for next generation wireless systems". IEEE communications magazine, vol. 52, n. 2, 186-195. 2014. DoI: 10.1109/MCOM.2014.6736761.

[9] Riadi, A., Boulouird, M., \& Hassani, M. M. R. "An Overview of Massive-MIMO in 5G Wireless Communications". In Colloque International TELECOM (pp. 10-12). 2017, May.

[10] Marzetta, T. L. "Massive MIMO: an introduction". Bell Labs Technical Journal, vol. 20, 11-22. 2015. Dor: 10.15325/BLTJ.2015.2407793.

[11] Ed. Wei Xiang, Kan Zheng, Xuemin (Sherman) Shen, "Part of: 5G Mobile Communications", pp. 77-116. ISBN: 978-3-319-34206-1. 2017. DoI: 10.1007/978-3-319-34208-5_4.

[12] Belhabib, M. "Investigation on radio channel over the air emulation by multi-probe setup" (Doctoral dissertation, Université Rennes 1). 2017.

[13] 3GPP TR, "5G; Study on channel model for frequencies from 0.5 to $100 \mathrm{GHz}$, ETSI, 3GPP TR 38.901 version 14.0.0 Release. 2017.

[14] 3GPP TR, "5G-NR-User Equipment (UE) radio access capabilities", ETSI, 3GPP TS 38.306 version 15.3.0 Release 1.2018.

[15] Ladvánszky, J. "Detection of 2x2 MIMO signals". INFOCOMMUNICATIONS JOURNAL, vol. 12, n. 3, 24-30. 2020. DoI: $10.36244 / \mathrm{ICJ} .2020 .3 .4$

[16] Csathó, B. T., Horváth, B. P., \& Horváth, P. Modeling the nearfield of extremely large aperture arrays in massive MIMO systems. INFOCOMMUNICATIONS JOURNAL, vol. 12, n. 3, 39-46. 2020. DoI: 10.36244/ICJ.2020.3.6.

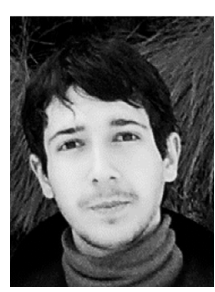

John Baghous obtained license degree in Telecommunication and electronic Engineering, master degree in Advanced Telecommunication Engineering, from Faculty of Engineering, Damascus University, lecturer at the university. Interested in all types of telecommunication systems especially mobile (4G, 5G ...etc.), wireless, WLAN, Wi-Fi, Li-Fi. Bluetooth etc. 